\title{
Virtual Cockpit Instruments - How Head-Worn Displays Can Enhance the Obstacle Awareness of Helicopter Pilots
}

\author{
Johannes Maria Ernst, Lars Ebrecht, Bernd Korn
}

This document contains the accepted version of the article published in IEEE Aerospace and Electronic Systems Magazine, vol. 36, no. 4, pp. 18-34, 2021.

C 2021 IEEE. Personal use of this material is permitted. Permission from IEEE must be obtained for all other uses, in any current or future media, including reprinting/republishing this material for advertising or promotional purposes, creating new collective works, for resale or redistribution to servers or lists, or reuse of anv copvriahted component of this work in other works.

The final, published version is available from

https://ieeexplore.ieee.org/document/9397798

DOI: 10.1109/MAES.2021.3052304 


\title{
Virtual Cockpit Instruments - How Head-Worn Displays Can Enhance the Obstacle Awareness of Helicopter Pilots
}

\author{
Johannes Maria Ernst \\ johannes.ernst@dlr.de
}

\author{
Lars Ebrecht \\ lars.ebrecht@dlr.de
}

\author{
Bernd Korn \\ bernd.korn@dlr.de
}

\author{
German Aerospace Center (DLR) \\ Institute of Flight Guidance \\ Braunschweig, Germany
}

\begin{abstract}
The rise of augmented reality glasses and related technologies offers new possibilities for the human-machine interface design of future aircraft. Today, head-worn displays (HWDs) are mainly used by military pilots, for instance by helicopter crews for low-visibility operations close to ground and obstacles. Nevertheless, recent technological advances in this area allow the prediction that these systems could become available for more pilots in the future. This work presents a concept how state-of-the-art HWD symbology can be expanded to get even more out of the advantages of this technology. With so-called "virtual cockpit instruments" (VCIs), an HWD can show information which is conventionally rendered on panel-mounted displays. These VCIs can be imagined as virtual display screens which can be positioned freely around the pilot. Their major benefit is that they create many new options for the design of a flexible, situation-adaptive cockpit environment. This paper introduces the general concept and presents several options how such an approach can be put into practice. Here, the concept is applied to helicopter operations in offshore windparks. We implemented a VCI-adapted obstacle awareness display and assessed a set of positioning variants for the new VCI. Two simulator studies - with 11 and 7 participants - provide interesting insights on the realization of this concept. In addition to high subjective ratings, the VCI significantly increased the pilot's head-up, eyes-out time - an important measure for challenging maneuvers close to obstacles. Overall, this work illustrates a promising concept for the human-machine interface design of future cockpits and discusses its potentials and limitations.
\end{abstract}

\section{Introduction}

Driven by new technology, the appearance of flight decks changed considerably over the years [1]. The classic gauge instruments were replaced by wide-screen, flat-panel displays. Simultaneously, the conventional panel-mounted displays (PMDs) were complemented by modern head-up displays (HUDs). These can augment the pilot's out-the-window view with virtually overlaid flight guidance symbology. With its origins in the military sector, a related technology is also on the rise: helmet-mounted or head-worn displays (HMD, HWD) are essentially like a HUD attached to the pilot's head [2]. Their central advantage is that HWDs can augment the reality in every viewing direction. This is a major plus, especially for rotorcraft pilots who often look in other directions than the forward axis where HUDs are installed. The involved technologies are currently pushed by the consumer electronics industry, which continually develops better, lighter, and more affordable virtual, mixed, and augmented reality (VR, MR, AR) glasses.

In aviation, HWDs present various types of symbology. Simple forms are used for weapon aiming or simply show see-through versions of a primary flight display (PFD) [3]. More advanced systems make use of integrated head-tracking modules to superimpose the symbology in a visual conformal or scene-linked way onto the real world. Examples of such implementations include the famous "tunnel-in-the-sky" symbol sets, brown-/white-out landing symbology, as well as terrain and obstacle representations for missions in degraded visual environments (DVE) (cf. Sec. 2.3).

At the German Aerospace Center (DLR), we introduced a new type of HWD symbology [4]. The so-called "virtual cockpit instruments" (VCI) are designed to be used together with the existing symbol sets mentioned above. A VCI is a two-dimensional virtual screen that is created and projected into the pilot's view via an HWD. As illustrated in Fig. 2a, VCIs can be regarded as a virtual version of a conventional cockpit instrument. The freedom to choose the position and the size of the instrument independently of the available panel monitors creates great flexibility for the design of future flight decks.

Such an approach can generate benefits for many use cases. In this article, we describe how the VCI concept can be applied to assist helicopter pilots in confined area operations: in this case, a hoisting maneuver and a platform landing in an offshore wind park. Nevertheless, the paper will also show the general advantages of VCIs, which makes the work a good basis if one wants to extend the concept to other use cases in the future. It provides an overview of the VCI concept and summarizes our main findings from two simulator studies with 18 participants in total. Finally, we draw conclusions how this approach can be expanded and applied to other scenarios.

The work was conducted within the scope of the project "Development of Powerful and Efficient Avionic-Platforms for Fixed and Rotary Wing Aircraft" (AVATAR). The joint project comprised several industrial partners and research institutions. It was funded by the German Federal Ministry 

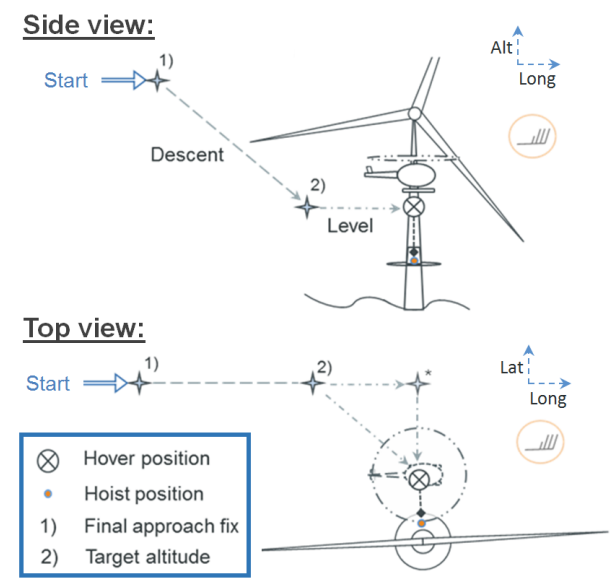

(a) Hoist operation at the lower access point of an offshore wind turbine. The maneuver requires hovering very close to the wind turbine tower.
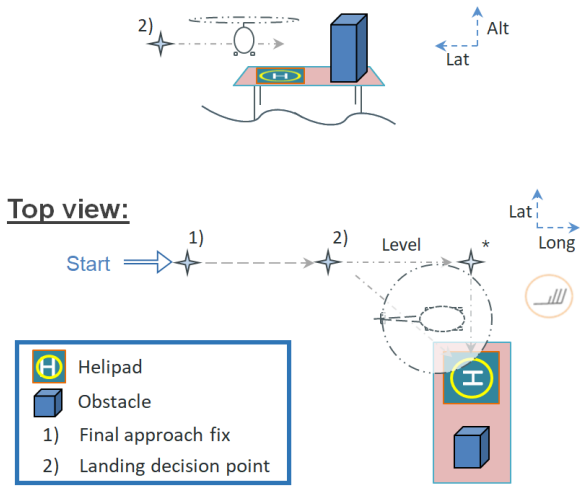

(b) Landing on an offshore platform with obstructions next to the helipad.

Figure 1 - Sketches of the offshore helicopter maneuvers considered for this evaluation.

of Economics and Energy in the frame of the national "Aeronautical Research Program V (LuFo V)”. A central project goal was the implementation and validation of future flight guidance applications using next generation avionics hardware in order to evaluate the potentials of future cockpit display systems. The paper is based on a series of conference publications [4]-[8].

\section{Background \& Related Work}

In this project, we applied our VCI concept to helicopter operations in offshore wind parks and implemented an obstacle awareness display as VCI. This section introduces the specifics of our use case and shows current obstacle awareness systems, which our implementation is based on. Finally, an overview of related work on HWD symbology is given.

\subsection{Helicopter Offshore Operations}

Helicopters are used in wind parks to transport workers and goods to offshore installations. This includes routine missions but also rescue and medical services under adverse conditions. The harsh weather, the small number of usable visual cues, and the fact that many maneuvers are conducted close to obstacles make these operations challenging for the crew. We provide a detailed review of helicopter offshore operations based on pilot interviews in a previous publication [9].

Figure 1a illustrates a maneuver to transfer persons between the helicopter cabin and the lower access platform of an offshore wind turbine. This is conducted if the upper hoist platform - which is easier to access - is not available. During this operation the pilot must hover at close distance to the wind turbine tower such that the hoist can be lowered to the personnel on the platform. As depicted in Fig. 1a, the pilot is additionally challenged by the fact that the turbine tower is located outside of the primary field of view, on the right side of the aircraft, at the pilot's three o'clock position.

Moreover, the pilots regularly land on offshore platforms and jack-up vessels. The helipads are often surrounded by various installations like cranes, towers or other structures. This can complicate the landing procedure and increase the risk of a collision. Figure $1 \mathrm{~b}$ shows such a landing procedure for a platform with a tower next to the landing deck. The final approach is conducted into the wind and ends at a landing decision point, which is located left and ahead of the helipad (point 2 in Fig. 1b). From this point, the pilot hovers sideways to land on the helipad.

\subsection{Obstacle Awareness and Warning Systems}

Obstacle awareness is crucial for offshore missions and helicopter operations in general. Hence, the industry has already developed obstacle awareness and warning systems (OAWS) which provide 360-deg near-field obstacle detection. Leonardo offers an EASA-certified obstacle proximity lidar system (OPLS), e.g. for their AW 139 helicopter. The system uses three lidar sensors to detect obstacles "as thin as a few $\mathrm{cm}$ " with $0.1 \mathrm{~m}$ accuracy and $25 \mathrm{~m}$ range [10]. Also, Airbus Helicopters presented flight tests of their prototypical rotorstrike alerting system (RSAS), which applies four commercial-of-the-shelf radar sensors to generate a 360-deg view [11]. Both systems have in common that they present the obstacle situation via an orthogonal top view on a head-down display. Both implemented a bi-modal human-machine interface (HMI) with visual and auditory cues. AgustaWestland applied a variable frequency tone and vocal announcements for warning and caution. Airbus Helicopters evaluated discrete tones, indicating the distance to the closest obstacle. Finally, the U.S. Army currently puts considerable effort into researching a complex tri-modal (visual, auditory, tactile) obstacle cueing system with speed-dependent threat level presentation [12]. The system comprises four radar sensors for the Black Hawk helicopter.

These developments show the relevance of obstacle detection technologies for future rotorcraft. However - from an HMI perspective - the question arises whether a head-up obstacle representation on an HWD might be preferable compared to a conventional head-down obstacle awareness and warning display (OAWD) as proposed by related work. Pilots prefer to look out the cockpit window paying attention to the surroundings, especially when operating in the vicinity of obstacles. Dividing the attention between the inside and the outside domain is distracting and generates additional workload. On the other hand, integrating a 360-deg top view into the HWD's native perspective may confuse the pilot, particularly if the top view is combined with visual conformal 3D symbology. 


\subsection{Head-Worn Display Symbology}

The key feature of HWDs is the presentation of additional information by superimposing symbology onto the pilot's natural out-the-window view. One of the most important HMI design paradigms in this context is the concept of "(visual) conformal symbology". The idea behind that is to show "synthetically generated symbols in spatial relation to real world objects" [13]. Also, the concept of "scene-linking" is closely related to this. The advantage of these concepts is that information from the display domain can be directly connected to the far domain. A well-established example of conformal symbology is the so-called tunnel/pathway/highway-in-thesky, which guides the pilot along the desired flight path even if the outside vision is degraded. Numerous publications have shown that this improves flight path tracking significantly (e.g. [14]). Further, scene-linked symbols are commonly used to highlight waypoints, obstacles, or other points of interest [13].

Helicopter flights in DVE are a major field of application for conformal HWD symbology. The scenarios include general low-visibility conditions due to adverse weather (fog, clouds, rain, darkness) as well as rotorcraft-specific scenarios like brownout/whiteout landings (where swirled-up particles degrade the pilot's out-the-window view). In such conditions, an HWD can synthetically provide missing outside visual cues and thereby increase the situation awareness of the crew [15]. Within their DVE Mitigation (DVE-M) program, the U.S. Army put great effort into the development of an enhanced vision system for their helicopters. Over the years they advanced their initial BOSS display [16] to a cutting-edge assistance system with several aircraft-mounted sensors and sophisticated guidance algorithms. Beyond visual conformal HWD and PMD symbologies, their system offers also tactile and auditory cueing [12], [17], [18]. The display format includes a conformal obstacle and landing zone presentation but combines this with a 2-D top view overlay. This 2-D domain incorporates many guidance elements like the target landing position ("dog house" symbol) and a horizontal velocity vector [19].

Another notable development in this area is the SFERION ${ }^{\circledR}$ system by Hensoldt [20]. Based on sensor and database information, the HWD shows a conformal terrain grid and contour lines to avoid controlled flight into terrain. Further, a conformal 3-D landing symbology provides necessary cues for low-visibility landings [21]. During a European DVE-M campaign, a joint team of researchers from DLR and Hensoldt demonstrated an HWD-based tunnel guidance system which was dynamically updated via a lidar sensor scanning the environment for obstacles [22]. Additionally, Schmerwitz et al. [23] developed DLR's own conformal approach and landing symbology. Its distinctive feature is the drift visualization via the pilot's peripheral vision. Finally, it should be noted that this list is not exhaustive as many other corporations and research institutes developed similar systems (e.g. [24], [25]).

Despite all benefits, the presentation of obstacle information as conformal overlay entails two major issues: First, the 3-D perspective comes with an uncertainty of locations and distances ("line of sight ambiguity" [26]). Second, the egocentric perspective is incomplete since the pilots cannot see what is behind them. Especially when operating in confined areas like urban spaces or near offshore constructions, obstacles can be located behind the helicopter, out of the pilot's view. This may cause hazardous situations where tail or rotor strikes can occur [9], [12]. Hence, this paper proposes the additional integration of an orthogonal, 360-deg top view with obstacle information into the HWD.

\section{Virtual Cockpit Instruments for Obstacle Awareness \& Collision Avoidance}

This work shows how existing HWD symbol sets can be enhanced by adding VCIs. Figure 2a shows an exemplary implementation: a conventional head-up PFD symbology is complemented by a virtual navigation display that is also generated by the HWD. Information that is typically displayed on conventional PMDs is thereby moved to the HWD. In Sec. 3.3, the depicted symbol set is further expanded by DLR's visual conformal landing symbology.

\subsection{Virtual Cockpit Instruments}

The prime advantage of VCIs is their independence from the flat panel screens of the cockpit. Conventional cockpit instruments are bound to the location, the size, and other specifications of the panel display rendering them. In contrast, a VCI can be created anywhere in the virtual space around the pilot. Its size and position can be adapted according to the requirements of the present task or flight phase. If it is currently not required, it can simply be hidden, which avoids clutter and clears the pilot's vision for the relevant information. If more display area is needed, the VCI can easily be enlarged or an additional VCI can be activated; options that are not available on a conventional flight deck with its inflexible panel display setup. This creation of additional display space is especially relevant for small helicopters like the ones often used for rescue medical services and in offshore wind farms because they have a very limited number of PMDs and cannot easily be retrofitted due to space constraints.

This great freedom and flexibility leads to the question: Where should such a virtual instrument be placed so that it creates a benefit for the pilot? Naturally, the answer to that question highly depends on the application scenario and on the actual VCI display contents. Having that in mind, we will start by presenting a comprehensive set of possible VCI positioning modes. In the rest of the paper, these concepts will then be applied to helicopter offshore scenarios in order to answer the stated question - for this specific case.

First of all, the display designer must choose a suitable frame of reference for the VCI. Conventional PMDs are - for obvious reasons - always fixed to the aircraft frame. Their position relative to the aircraft does not change; they are "aircraft-fixed". If the pilot looks somewhere else, the displays get out of sight. In a previous publication [4], we also implemented the virtual instruments like this. However, the VCI approach offers more options: a VCI can also be attached to the head-fixed frame of reference. This means that it will strictly follow the pilot's head movements and always remain in sight (cf. Fig. 2b). Figure 3 provides a schematic overview of the developed VCI positioning options, including these two basic variants (Aircraft-Fixed, Head-Fixed) but also combinations and derivatives of them (Aircraft-Related, Mixed). For example, the VCI can primarily have an aircraft-fixed position but every time the pilot looks to the side, the VCI becomes head-fixed to remain visible. We call this the Mixed mode. Finally, the Aircraft-Related mode has a similar switch- 


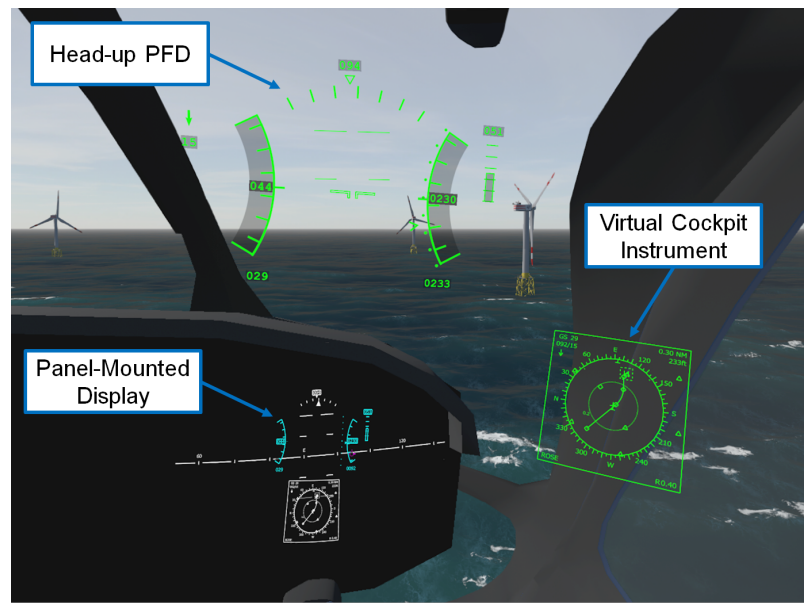

(a) An aircraft-fixed VCI during the approach phase: the VCI is positioned right of the instrument panel. The image also shows the head-up PFD and the symbology displayed on the PMD (PFD \& OAWD). This display setup corresponds to the display variant VCI-Mixed in study II.

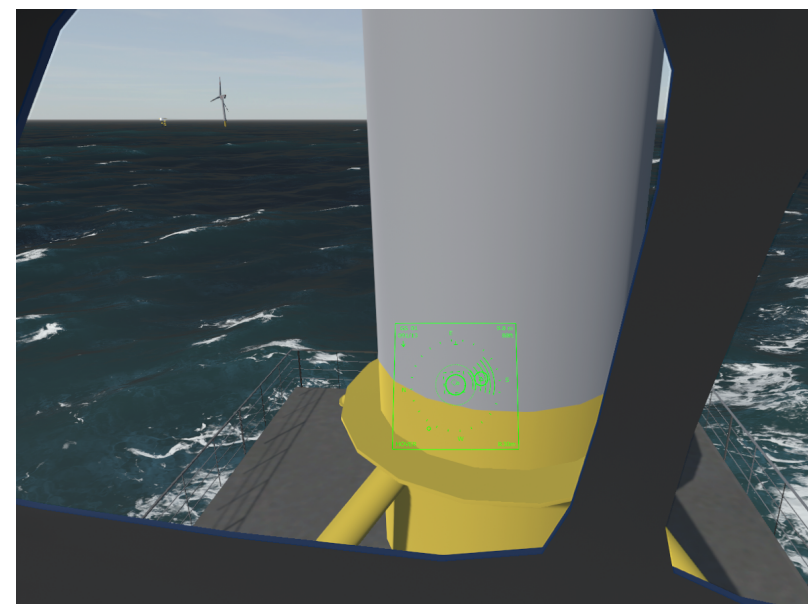

(b) A head-fixed VCI during hover: the VCI follows the pilot's head movements. Here, the pilot looks to the right towards the wind turbine. The VCI remains within the field of view. This corresponds to the following experiment conditions: Mixed-HDD-Below and HeadFixedBelow in study I as well as VCI-HeadFixed and VCI-Mixed in study II.

Figure 2 - Illustrations of selected $\mathrm{VCl}$ modes during different phases of an approach and hover maneuver at an offshore wind turbine. The images were generated on VR goggles (study I) and demonstrate the pilot's view through a monochrome green, see-through HWD (study II). Details about the studies and the various display variants are given in Sec. 4.
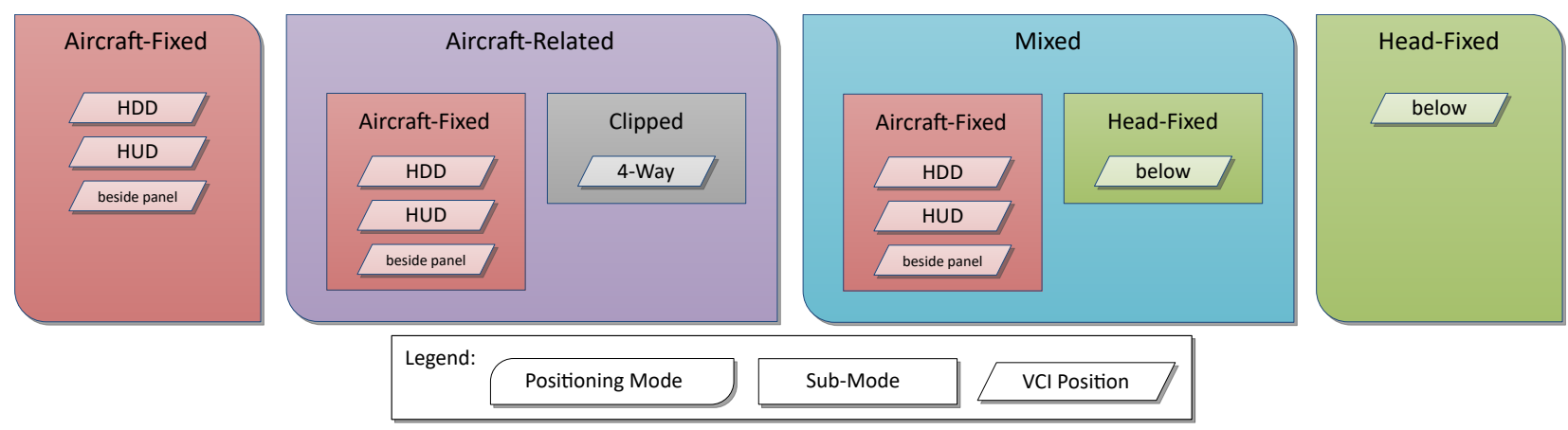

Figure 3 - Overview of the four VCI positioning modes (Aircraft-Fixed, Aircraft-Related, Mixed, Head-Fixed) and the respective instrument positions. Aircraft-Related and Mixed comprise sub-modes depending the pilot's viewing direction.

ing behavior between two sub-modes. However, instead of the "head-fixed" sub-mode, it features a "clipped" state. Here, the VCI appears at one of the four borders of the field of view (FOV).

As shown by the rhomboids in Fig. 3, the VCI can - of course - have different positions within the chosen frame of reference. Within the aircraft-fixed frame, the straightforward way is to place the VCI where a conventional cockpit instrument would be, i.e. in the panel area. This variant is called "HDD" (head-down display). Furthermore, we implemented a "HUD" position. Here, the VCI is located above the instrument panel in the windshield area where a conventional HUD would be mounted. In addition to these familiar options, we also placed the VCI on the right of the instrument panel ("beside panel"). Fig. 2a shows how this mode appears to the pilot looking through the HWD. Regarding the headfixed position, we realized only one position where the VCI is located "below" the pilot's viewing direction (i.e. in the lower center of the HWD screen). Figure $2 \mathrm{~b}$ illustrates this variant during an offshore hover maneuver. More information on these positioning options can be found in [6].

\subsection{Obstacle Awareness and Collision Avoidance Symbology for the VCI}

Inspired by the display formats presented by Airbus and AgustaWestland (cf. Sec. 2.2), we implemented a VCI-adapted obstacle awareness and warning display (VCI-OAWD). It was specifically customized for the usage as a VCI on a seethrough display. Further, it was adapted to the monochrome green color of DLR's flight-certified HWD. Figure 4 shows the two pages of our VCI-OAWD. The approach page is similar to a conventional navigation display in rose mode. It shows the approach route, surrounding objects and various supplemental information like the current wind conditions et cetera. The hover page is activated when the helicopter passes a certain distance to the desired hover position. It provides an orthogonal $360^{\circ}$ top view of the near field including the desired hover position and nearby obstacles. To improve the pilot's distance estimation, it renders two circles indicating the main rotor size and the required safety clearance. Similar to Airbus' RSAS, the area around the ownship is divided into sectors which are highlighted if an obstacle is detected within that zone. 


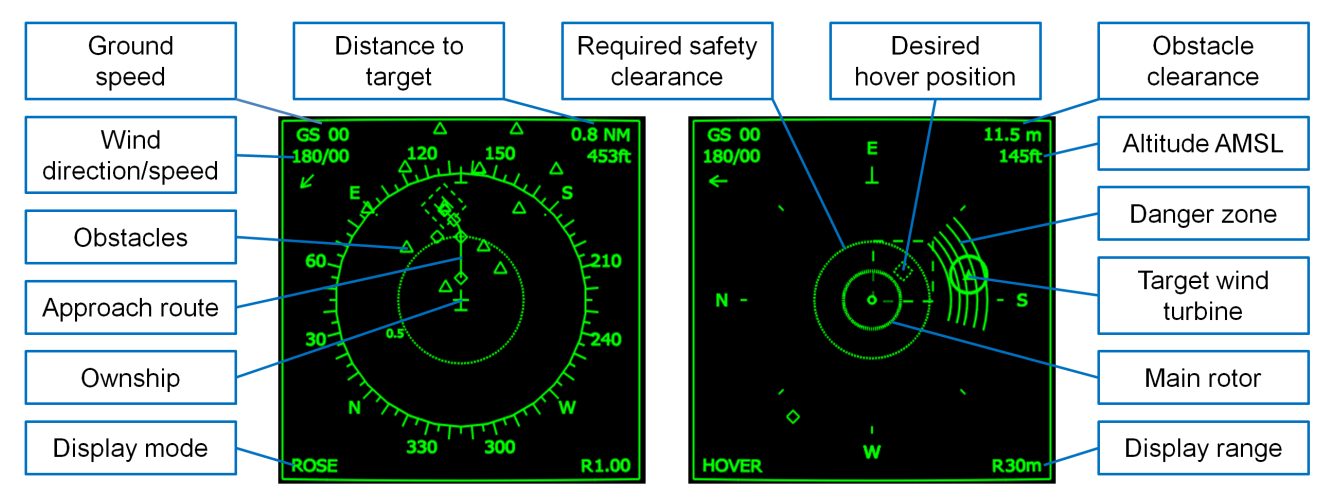

Approach Page

Hover Page

Figure 4 - The two pages of the developed obstacle awareness symbology for the $\mathrm{VCl}$. The display switches from the approach to the hover page when passing a certain distance to the desired hover position. The black background corresponds to transparent areas in the see-through HWD.

\subsection{Integration with State-of-the-Art Head-Up Symbology}

As described in Sec. 2.3, a typical HWD is usually filled with various kinds of symbology. Thus, it is an integral part of our work to investigate how the proposed VCI concept works together with already existing symbol sets. To do so, we integrated the new VCI-OAWD with a standard head-up PFD and with DLR's proven landing symbology [23]. Figure 5 illustrates how the whole system appears to the pilot during a landing maneuver on an offshore platform. The conformal landing symbology (CLS) was initially developed for approaches and landings in DVE. It comprises a contact analog circle highlighting the desired touchdown position. Further, a rectangular frame provides a reference that is still in sight when the pilot hovers directly above the landing position. This reference frame supports the rough positioning and gives additional attitude cues. Two bracket symbols are used for the precise longitudinal positioning. The desired landing spot is reached when the brackets are aligned with the gap in the front edge of the rectangular frame. In addition, a ball indicates the precise longitudinal and lateral deviation relative to the center of the forward edge of the virtual landing pad. It is additionally stimulated with a prediction from the current ground speed. Details on the development and evaluation of this symbology are given by Schmerwitz et al. [23].

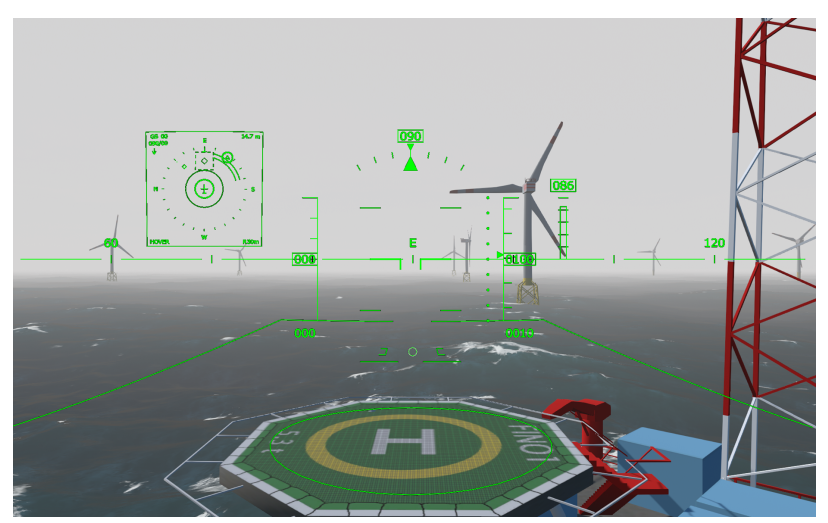

Figure 5 - The pilot's view during the landing maneuver with the superimposed symbology generated by the monochrome green JEDEYE ${ }^{\mathrm{TM}}$ helmet. The symbol set comprises a head-up PFD (center of the image), DLR's visual conformal landing symbology (lower center), and the developed VCI-OAWD (upper left).

\section{Evaluation Method}

We conducted two simulator studies to assess our VCI implementation. This section describes the methods of both experiments.

\subsection{Research Questions}

The overall research goal for both evaluation studies was to get initial feedback on our novel VCI-OAWD. The intent was to investigate if our approach is a useful addition to stateof-the-art flight guidance displays. Moreover, we wanted to gather suggestions for possible improvements of the symbology.

Study I As discussed in Sec. 3.1, VCIs can be positioned in many ways. Thus, the focus of the first experiment was on the selection of the most promising positioning options. Further, we tested different VCI sizes and had a look at the interaction with the head-up PFD symbology. The condensed number of feasible VCI variants and all other findings formed the basis for our second study.

Study II For the second evaluation, we further improved our VCI implementation based on the initial feedback. The first study was conducted in a fully immersive VR setup where the pilot's view through the HWD was emulated with VR goggles (details below). This time, we assessed the pre-selected variants in an advanced simulation environment with DLR's flight-certified see-through HWD. Thereby, we wanted to confirm that the positive reception from the first study also holds true under these more realistic conditions.

\subsection{Participants}

Study I Eleven subjects (1 female, 10 male) with a mean age of 38 (between 26 and 61) took part in the first simulator study. All participants had experience with helicopter-flying, either in the simulator or in real flight. Three held a helicopter license (2 airline transport pilot license (ATPL), 1 commercial pilot license (CPL)), five held a fixed-wing license. The actual flight hours (without simulator hours) ranged from 0 to 6400 (mean: $1248 \mathrm{~h}$ ). Seven subjects had a mean experience of $56 \mathrm{~h}$ with HWDs. 


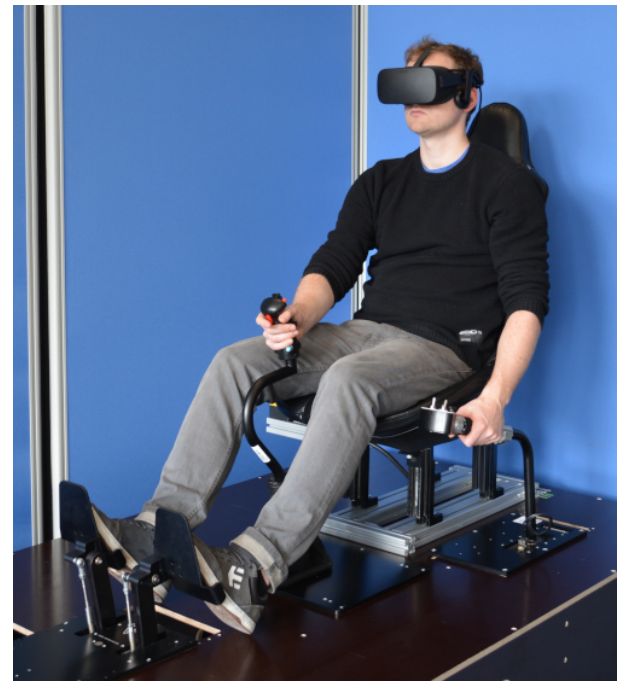

(a) Study I - Fully virtual setup with a pilot wearing the Oculus Rift VR goggles.

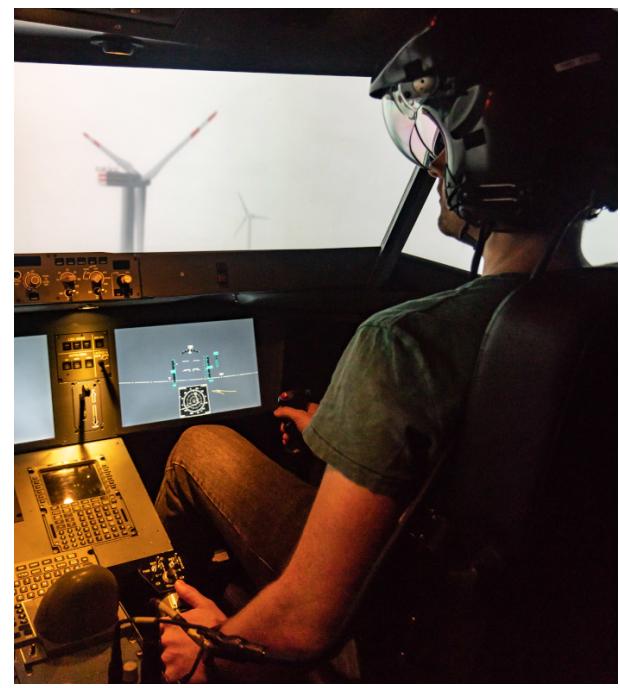

(b) Study II - Pilot wearing the JEDEYETM see-through HWD in the GECO simulator.

Figure 6 - Experimental setup of the two simulator studies.

Study II Seven male helicopter pilots with an average age of 46 (range from 38 to 62) participated in the second experiment. They had a mean flight experience of $1985 \mathrm{~h}$. One held a private pilot license (PPL), four a CPL, and two an ATPL. Five pilots reported prior HWD experience (mean: $108 \mathrm{~h}$ ). Three of them already participated in the first VCI study described above.

\subsection{Apparatus}

Study I The first experiment used a fully virtual setup. As pictured in Fig. 6a, the participants wore the Oculus Rift CV 1 VR goggles and steered the helicopter with professional, active force-feedback flight controls [27]. The VR HWD simulated the pilot's view through a see-through HWD as it is used on a flight deck. The virtual world comprised the surroundings, the cockpit, and the overlaid symbology (cf. Fig. 2a). The HWD had a resolution of $1080 \times 1200$ pixels per eye and a diagonal FOV of around $110^{\circ}$.

The flight mechanics were simulated by DLR's custommade EC135 flight model. It includes an automatic flight control system (AFCS) offering several upper modes [28]. For these trials, the cyclic axes were in "attitude command, attitude hold”, which enables direct control of pitch and roll attitude via the center stick. If the pilot let go of the cyclic, the helicopter returned to trimmed attitude. The yaw rate was commanded via the pedals being in "rate command, direction hold". Finally, height changes were issued via the collective. Each deflection of the collective led to a certain climb or sink rate. While in neutral position, the AFCS held the current altitude ("vertical velocity command, height hold").

Study II The follow-up experiment was conducted in DLR's Generic Experimental Cockpit (GECO). This fixed-base simulator provides a collimated outside vision with $180^{\circ} \times 40^{\circ}$ FOV. It has a highly modifiable flight deck which can be equipped with helicopter flight controls [27] as well as with our flight-certified see-through HMD.

For the evaluation of the VCI concept we used the Elbit JEDEYE $^{\mathrm{TM}}$. It is a binocular, optical see-through HMD with a wide FOV of $80^{\circ} \times 40^{\circ}\left(60^{\circ}\right.$ overlap). The resolution of the monochrome green image sources is $1920 \mathrm{px} \times 1200 \mathrm{px}$ per eye $(2200 \mathrm{px} \times 1200 \mathrm{px}$ in total). Correct spatial integration of the virtual symbology with the outside vision and the real world environment is ensured by an electromagnetic headtracking system. Figure $6 \mathrm{~b}$ illustrates the whole setup with the HWD, the PMD, and the offshore windpark presented on the projection system. As visible in the picture, the cockpit shell of the GECO replicates an Airbus A350, which leads to a restricted out-the-window view compared to most helicopters. Despite this limitation, the collimated outside vision and the integrated flight-certified HMD make this simulator a good choice for this experiment and the study a valuable step before the flight test with DLR's research helicopter.

The flight simulation was provided by X-Plane 11.41 using a tailor-made plugin. As aircraft model we applied the Eurocopter EC135 developed by Rotorsim [29]. It was customized to suit our needs with the help of the Rotorsim developer and DLR's test pilots.

\subsection{Task}

In both studies, the pilots had to conduct a hover maneuver at an offshore wind turbine (as introduced in Sec. 2.1). The second study comprised an additional landing task on an offshore platform.

Study I The participants had to fly an approach to an offshore wind turbine and perform a simulated hoist maneuver at its lower access point. The runs were started in-flight, approximately $0.8 \mathrm{NM}$ from the target position. The pilots had to fly a left turn towards the wind turbine and into the wind. As depicted in Fig. 1a, the final approach was a straight descent towards a point left of the wind turbine. From there, the final segment was a horizontal transition to the desired hover position. At that point, the wind turbine tower was located at the pilot's 3 o'clock position with half a rotor diameter clearance between the main rotor tips and the tower. As soon as they reached this position, they had to acknowledge "on position" by pressing the trigger button on the cyclic. From then on, the task was to hold this position as precisely as possible for $60 \mathrm{~s}$.

Study II The hover task of study II was similar to the one in the first study described above. Additionally, the pilots had to perform a landing maneuver on an offshore platform. 
Table 1 - Specifications of the display conditions tested in the two experiments. Each line of the table corresponds to one display variant. The different colors are chosen to match the coloring of the results plots in the following chapter.

(a) Study I - Hover Task (subset of conditions relevant for this paper).

\begin{tabular}{ccccc}
\hline \multirow{2}{*}{$\begin{array}{c}\text { HWD } \\
\text { Symbology }\end{array}$} & Mode & \multicolumn{2}{c}{ VCI } & PMD \\
\cline { 2 - 4 } & & Aircraft-Frame & Head-Frame & Symbology \\
\hline PFD + VCI & AC-fixed & HDD & - & - \\
PFD + VCI & Mixed $^{\text {a }}$ & HDD & below & - \\
PFD + VCI & Head-Fixed $^{\text {Helion }}$ & - \\
\hline
\end{tabular}

a VCI position changes based on the pilot's line of sight.

(b) Study II - Hover Task.

\begin{tabular}{|c|c|c|c|c|}
\hline \multirow{3}{*}{$\begin{array}{c}\text { HWD } \\
\text { Symbology }\end{array}$} & \multicolumn{3}{|c|}{ VCI } & \multirow{3}{*}{$\begin{array}{c}\text { PMD } \\
\text { Symbology }\end{array}$} \\
\hline & \multirow[t]{2}{*}{ Mode } & \multicolumn{2}{|c|}{ Position } & \\
\hline & & Aircraft-Frame & Head-Frame & \\
\hline PFD & - & - & - & \multirow{3}{*}{$\sum_{0} \otimes \stackrel{\theta}{a}$} \\
\hline $\mathrm{PFD}+\mathrm{VCI}$ & Head-Fixed & - & below & \\
\hline $\mathrm{PFD}+\mathrm{VCI}$ & Mixed $^{\mathrm{b}}$ & beside panel & below & \\
\hline
\end{tabular}

${ }^{b}$ VCI position changes based on the flight phase.

(c) Study II - Landing Task.

\begin{tabular}{ccccc}
\hline \multirow{2}{*}{$\begin{array}{c}\text { HWD } \\
\text { Symbology }\end{array}$} & Mode & \multicolumn{2}{c}{ VCI } & PMD \\
\cline { 2 - 4 } & & Aircraft-Frame & Head-Frame & Symbology \\
\hline PFD & - & - & - & - \\
PFD + CLS & - & - & below \\
PFD + VCI & Head-Fixed & - & below \\
PFD + CLS + VCI & Head-Fixed & - & \\
\hline
\end{tabular}

Similar to the hover task, they conducted an approach to a landing decision point left of the helipad. From there, they hovered sideways onto the helideck while avoiding the obstacle located beside the touchdown zone (see Fig. 1b).

\subsection{Experimental Design \& Tested VCI Variants}

Both studies were designed as a within-subject experiment.

Study I Our first study comprised 14 test conditions and three independent variables. The details of the full test matrix are explained in [6]. In this paper, we focus only on the three most promising display types. An overview of this selection is given in Table 1a. In all conditions, the PMDs were switched off and the head-up PFD and the VCI on the HWD were the only flight guidance symbology available.

The tested variants differed in their VCI positioning mode (cf. Fig. 3). In AircraftFixed-HDD, the VCI was at a fixed position relative to the aircraft frame of reference. In HeadFixedBelow, the VCI was rigidly coupled to the pilot's head frame of reference. Mixed-HDD-Below represented a mixture of both pure variants. In this Mixed mode, the VCI changed its position based on the pilot's line of sight (LOS). Whenever the pilot looked in the direction of the VCI's aircraft-fixed position, the VCI remained stable at this position. As soon as this aircraft-fixed VCI left the pilot's FOV, the VCI jumped to its head-fixed position. This ensured that the VCI was always available even if the pilot turned the head away from the instrument panel. Compared to the head-fixed option - where the VCI moves with every head rotation -, the mixed variant is more steady and visually less disturbing as long as the pilot has the fixed VCI within the FOV.

As specified in Table 1a, the aircraft-fixed VCI was always located at the head-down display (HDD) position, which means that the instrument appeared virtually on top of the inactive PMD screens. The head-fixed VCI was positioned below the pilot's viewing direction, in the lower central area of the FOV. Figure $2 \mathrm{~b}$ illustrates how the head-fixed position looked like.

Study II In the follow-up study we had different sets of display variants for the two tasks. In addition to the display type, we included a second independent test variable: wind condition. It comprised two wind strength levels: 10 knots and 25 knots, both from $90^{\circ}$ (corresponding to head-wind during hover and landing).

Hover Task: The hover task involved three independent display conditions, which were characterized by the type of VCI tested on the JEDEYETM helmet: 1) no VCI, 2) head-fixed VCI, and 3) mixed VCI. As shown in Table 1b, the HWD additionally displayed a head-up PFD in all conditions. Also, the PMD showed the same information throughout the whole experiment: a colored, head-down version of the developed symbol set including the OAWD and the PFD (see Figs. 2a and 6b). The first test condition (line 1 in Table 1c) represented the experiment baseline with the OAWD only visible on the PMD. Thus, this test condition will be referred to as $P M D-O A W D$. Both other conditions included the VCI-OAWD in two different positioning modes. The first, VCI-HeadFixed, was identical to the 

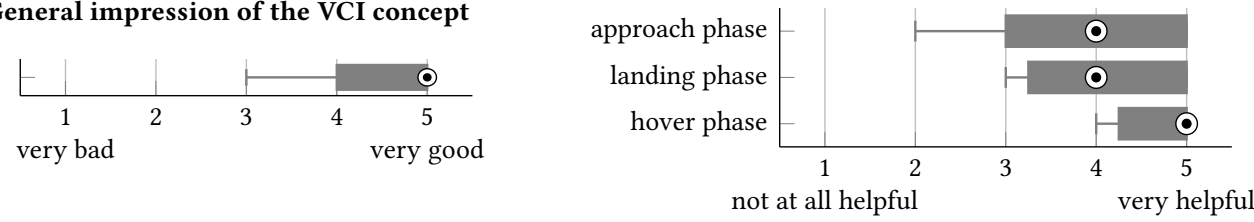

Figure 7 - Overall rating of the Virtual Cockpit Instruments. ${ }^{1}$

display tested in the first study (see Fig. 2b). The second, VCIMixed, was a "mixed" variant, where the position of the VCI changed during the flight. Here, it changed from an aircraftfixed position right of the instrument panel (see Fig. 2a) to an head-fixed position similar to VCI-HeadFixed (see Fig. 2b). In contrast to the first study, however, the position changes were not triggered by the pilot's viewing direction. Instead, it only changed once at the transition between the approach and the hover phase. This means that during the $60 \mathrm{~s}$ hover maneuver, the symbology of VCI-Mixed and VCI-HeadFixed was identical.

Landing Task: The focus of the landing evaluation was the integration of the VCI with state-of-the-art HWD symbology. Thus, we tested different combinations of DLR's visual conformal landing symbology, the head-up PFD, and one selected version of the VCI (HeadFixed-Below). Table 1c shows the resulting display conditions: 1) PFD, 2) PFD + CLS, 3) PFD + VCI, and 4) PFD + CLS + VCI. The PMD symbology showed the same symbol set as during the hover task and was not varied between the conditions.

\subsection{Procedure}

At the beginning of both experiments, the pilots received a comprehensive introduction and briefing. Thereafter, we conducted several training flights for the subjects to get accustomed to the simulator, the tasks, and the different symbol sets. After a break - during which the participants filled out a biographical survey - we started the testing phase.

Study I As detailed in [6], all pilots had to fly 14 test conditions (in counterbalanced order). After each run, they participants filled out a short questionnaire. At the end of the experiment, they also completed a debriefing form. The total study duration ranged from $2.5 \mathrm{~h}$ to $4 \mathrm{~h}$.

Study II The testing phase was split into two blocks. In one block the participants flew the six hover maneuvers described above ( 3 display $\times 2$ wind conditions). The other block comprised eight landings on an offshore platform (4 display $\times 2$ wind conditions). The order of the task blocks as well as of the display and wind conditions was counterbalanced between the participants. A break was scheduled between the two blocks. The subjects completed several tailormade questionnaires: a post-flight questionnaire, post-block surveys containing specific questions for the two major parts of the study, and a final debriefing questionnaire. In total, the experiment took about $4 \mathrm{~h}$.

\subsection{Dependent Variables}

Several dependent measures were recorded during both studies. Regarding objective data, we investigated the influence of the display conditions on the pilots' head motion patterns and on the flight performance. The latter includes the accuracy of the hover maneuver (study I+II) as well as the precision and obstacle clearance during the landing task (study II). Further, we collected subjective feedback on various aspects of the VCI concept: 1) comparison of VCI and PMD, 2) integration with state-of-the-art head-up symbology, 3) VCI size, readability, and clutter, 4) comparison of VCI positioning options.

\section{Evaluation Results}

This section provides a summary of the most important findings of both evaluation studies. First, we will review the pilots' feedback on various aspects of the VCI concept. Second, we will assess the effects of the tested display conditions on the flight performance. Finally, we will check how the VCI influences the pilots' head motion patterns. The section is based on a number of conference papers [6]-[8], which one can refer to for further results.

\subsection{Pilot Feedback on the VCI}

Overall, the VCI concept was received very positively. In the second study, all pilots except one stated that their general impression of the VCI concept is "very good" or "good" (cf. Fig. 7). Accordingly, many pilots assessed the value of the VCI as "(very) helpful". As can be seen in Figure 7, the VCI seemed to be most helpful during the hover phase. For the approach and the landing phase, the VCI received lower but still (very) positive ratings.

\subsubsection{Virtual Instrument vs. Panel-Mounted Display}

A remarkable result is that all pilots preferred to have the hover symbology as a VCI instead of having it on a conventional PMD. This also matches the subjects' overall conclusion on the three display conditions they tested with the JEDEYE ${ }^{\mathrm{TM}}$. Figure 8a shows that both variants containing the VCI were rated significantly better than the baseline $P M D-O A W D$ condition, where the OAWD was displayed only on the PMD and the head-up symbology comprised a PFD only. The median overall rating for both VCI conditions was "good". However, while almost all pilots agreed on their rating of VCI-HeadFixed, the results of VCI-Mixed show noticeable disagreement between the participants. The good VCI ratings go in line with all participants agreeing to the statement that "in the future, more information that is currently presented on conventional PMDs should be displayed on the HWD."

Figure $8 \mathrm{~b}$ depicts the pilots' estimation of how much they used the PMD compared to the HWD symbology. Obviously, since the baseline condition showed only a head-up PFD without further hover/obstacle information on the HWD, the participants looked down to the PMD very often. The

\footnotetext{
${ }^{1}$ Boxplots show median (dot/circle), 25th and 75th percentiles (filled rectangle), and outliers (x markers) with whisker length $1.5 \mathrm{IQR}$.
} 


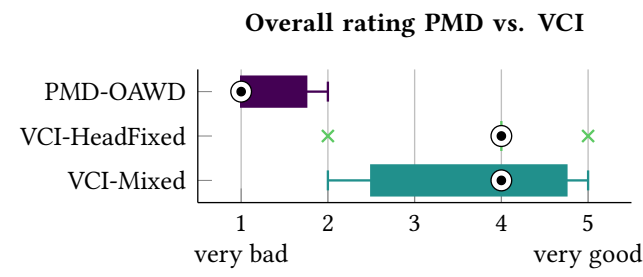

(a) Overall rating of the presented display sets.
Usage ratio HWD vs. PMD

symbology during hover

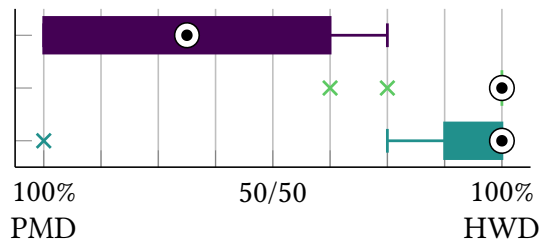

(b) Usage ratio head-up vs. head-down symbology.

Figure 8 - Comparison of the PMD-based vs. the VCl-based OAWD in the JEDEYE ${ }^{\mathrm{TM}}$ study. ${ }^{1}$

median head-down usage ratio was approximately $70 \%$. On the contrary, the VCI drastically decreased the head-down time to almost zero.

\subsubsection{Integration with State-of-the-Art Head-Up Symbology}

Usually, an HWD is not used exclusively to display a VCI. As described in Sec. 2.3, it typically shows a head-up version of a PFD as well as various kinds of visual conformal flight guidance symbology. Therefore, our experiments also checked how these different types of displays can be integrated with each other.

In the first study, we showed that a state-of-the-art head-up PFD works together with the VCI [6]. The focus of the second study was on the integration of the VCI-OAWD with DLR's conformal landing symbology. Figure 9 shows the pilots' reception of the different combinations of VCI and CLS that we tested. As expected, the results confirm the value of the CLS: the PFD-CLS condition was rated better than the baseline (PFD only) and also better than the PFD- $\mathrm{VCI}$ variant. The most important result, however, is that the participants favored the simultaneous inclusion of both symbol sets (PFD-CLS-VCI). The median rating of this symbology was "very good" even though the combined setup seems quite complex at first.

Overall rating of the different display conditions

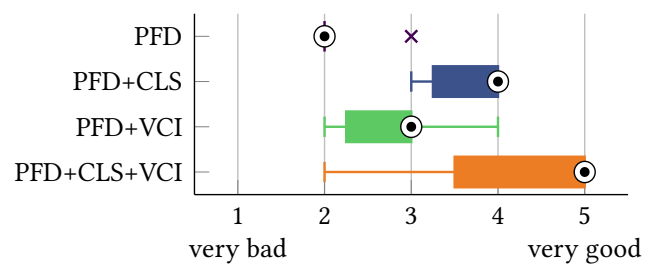

Figure 9 - Overall rating of the presented display sets. ${ }^{1}$

\subsubsection{VCI Size, Readability, and Clutter}

In the VR-based study I we compared three different VCI sizes. All subjects agreed that $14 \times 14$ degrees was a proper size for the VCI. Of course, the selection of a feasible size depends largely on the VCI contents and the resolution of the HWD. On the Oculus Rift CV 1, this size corresponds to a display area of approximately $194 \times 194$ pixels [30]. On the JEDEYETM with its higher angular resolution, the VCI size was reduced to approximately $10 \times 10$ degrees or $270 \times 270$ pixels. The majority of pilots in this second study indicated that this was a good size. A few would prefer to slightly increase the VCI.

The virtual instruments are see-through displays where the symbology is superimposed onto the reality. Therefore, the readability of the VCI is not only a matter of size and resolution but also of the instrument's background. Depending on the VCI positioning mode, the background can be uniform and constant or heterogeneous and ever-changing.

The first study compared several positioning options. It found that the reality behind the VCI had a strong negative impact on the readability when the VCI was located at the HUD position. The subjects explained that they had major problems reading the symbology since the horizon crossed the VCI in the background. The differences between the dark ocean in the lower half and the bright sky in the upper half of the display caused strong contrast issues. On the contrary, the VCI projected onto the dark instrument panel (AircraftFixed-HDD) performed best. Nevertheless, the most important finding was that all head-coupled variants caused no or only weak readability issues - despite the fact that the instrument background was continually changing during head movements.

\section{Impact of the underlying reality on the readability of the VCI}

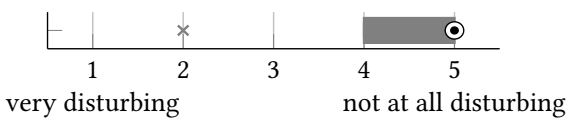

Figure 10 - Readability of the Virtual Cockpit Instrument on the JEDEYETM 1

A major question of the second experiment was whether the good readability of the head-coupled variants on the VR goggles could be confirmed with an actual see-through HMD. As illustrated in Fig. 10, this can be answered with yes: The participants acknowledged that the underlying reality had no negative influence on the readability of the VCI.

The pilots are not only interested in reading the VCI but also want to monitor the real world. Thus, it not enough to guarantee that the underlying reality does not degrade the readability of the VCI. Vice versa, we also have to ensure that the VCI being overlaid onto the reality does not disturb the pilots' out-the-window view. The participants reported that the VCIs did not clutter the natural vision in most conditions. Only the VCI positioned in flight direction above the panel (AircraftFixed-HUD) masked too much of the central, forward FOV and disturbed the pilots' out-the-window view during the approach.

In summary, the results of both studies show that the VCI size and position should be chosen carefully because this may have strong effects on readability and clutter. The setups for study II (Mixed, Head-Fixed) were found to be reasonable choices in that matter. 
Reception of the different VCI behavior during approach (aircraft-fixed) and hover (head-fixed)

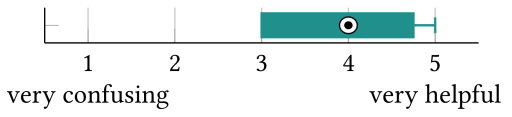

Satisfaction with the aircraft-fixed VCI position beside the instrument panel

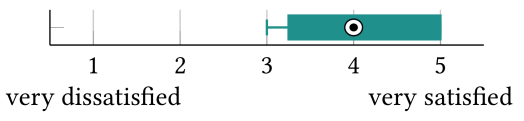

Figure 11 - Rating of the VCI positioning mode Mixed in the JEDEYETM ${ }^{\mathrm{T}}$ study. ${ }^{1}$

\subsubsection{Comparison of VCI Positioning Options}

As described in Sec. 3, a major benefit of VCIs is their flexibility regarding position and frame of reference. In our first VR-based study, we tested a large number of possible positioning options and selected the most promising variants. The details of this assessment are presented in [6]. In summary, the conditions Mixed-HDD-Below and HeadFixed-Below were the preferred variants, closely followed by the more conventional AircraftFixed-HDD mode. Consequently, the follow-up study took a closer look at these pre-selected positioning options. The remainder of this results chapter also focuses only on these most relevant VCI modes.

The pilots tested two different positioning modes for the VCI on the JEDEYETM. In the VCI-HeadFixed condition, the VCI was head-fixed throughout the whole flight. It remained inside the pilots' FOV all the time. By contrast, in the VCIMixed variant, the VCI was located at an aircraft-fixed position beside the instrument panel during the approach phase. As soon as the pilot transitioned to the hover phase, the VCI switched to a head-fixed position similar to VCI-HeadFixed. When asked about their preferred positioning mode, two pilots chose VCI-HeadFixed, three favored VCI-Mixed, and the remaining two stated that both are equal.

To further assess the Mixed mode, the participants were queried on the helpfulness of the altering VCI behavior in the two phases of the hover task. Figure 11 confirms that this was seen as helpful. Also, the VCI position that we chose for the approach phase - right of the instrument panel (cf. Fig. 2a) was satisfying for the majority of the pilots.

\subsection{Flight Performance}

In addition to the pilots' subjective ratings, we also evaluated which effects the VCI had on the flight performance. First, we looked at the accuracy of the hover maneuver that the participants performed in both studies. Second, we checked the performance achieved during the landing maneuver, which was only conducted in the second experiment.

\subsubsection{Hover Accuracy}

The pilots' task during the hover maneuver was to hold the target position as precisely as possible for $60 \mathrm{~s}$. The achieved positional accuracy is illustrated for both studies by means of two graphs respectively: First, we checked the horizontal flight paths during the hover via a top view. Second, we computed the length of the track that they covered within these $60 \mathrm{~s}$. The corresponding plots are given in Figs. 12 and 13 .

Study I - VR-Goggles with Normal Out-the-Window View Overall, the flight paths of the different display conditions in study I appear to be alike. The top views in Fig. 12a show good and similar positional accuracy for all variants.
Also, the boxplots of the covered track give a uniform picture. However, with AircraftFixed-HDD the participants seem to have lost control in two runs. This led to significantly longer covered tracks, large deviations, and even rotor strikes for these flights.

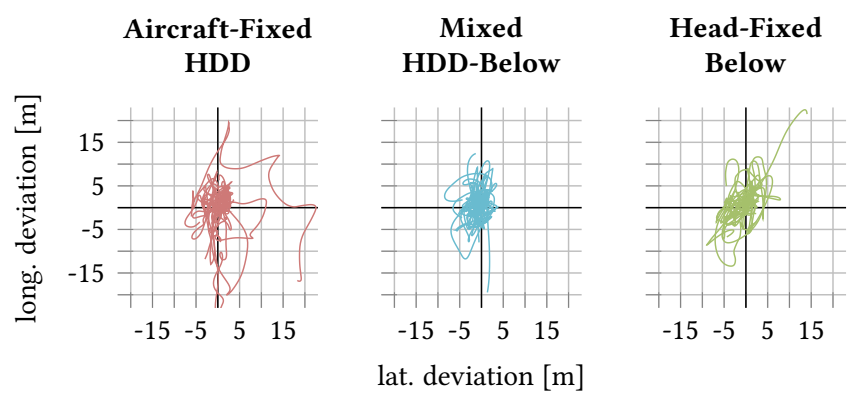

(a) Top view of the flight paths.

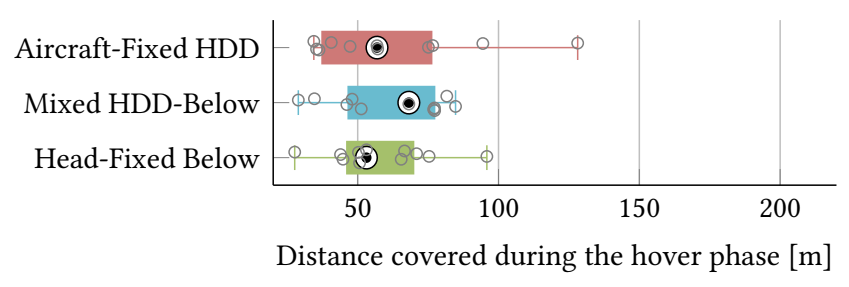

(b) Covered track.

Figure 12 - Positional accuracy during the $60 \mathrm{~s}$ hover phase of the VR-based study I (selected display variants). ${ }^{2}$

Study II - JEDEYETM with Restricted Out-the-Window View As can be seen in Fig. 13, the positional accuracy observed in the second study was overall lower than with the fully virtual setup. The top views of all variants indicate that the flight paths covered a much wider area. Also, the predominant deviation direction seems to be left behind the desired hover position (in the third quadrant of the graph). The reason for that phenomenon is presumably that in this position the wind turbine tower was easier to observe from the cockpit: the obstacle then appears not at $90^{\circ}$ to the right but rather at around $45^{\circ}$. If we compare the three tested display conditions of the second study among each other, we find larger lateral deviations for $P M D-O A W D$ compared to both variants with the VCI. The pilots' difficulties in holding the desired hover position are also reflected in the track covered during the hover phase. Figure $13 \mathrm{~b}$ reveals that $P M D-O A W D$ caused longer tracks for several flights.

\subsubsection{Landing Performance}

Concerning the performance of the landing maneuver, we checked two aspects: landing precision and obstacle clearance. Figure 14 shows a top view of the locations where the

\footnotetext{
${ }^{2}$ Raw values are illustrated by gray circles.
} 


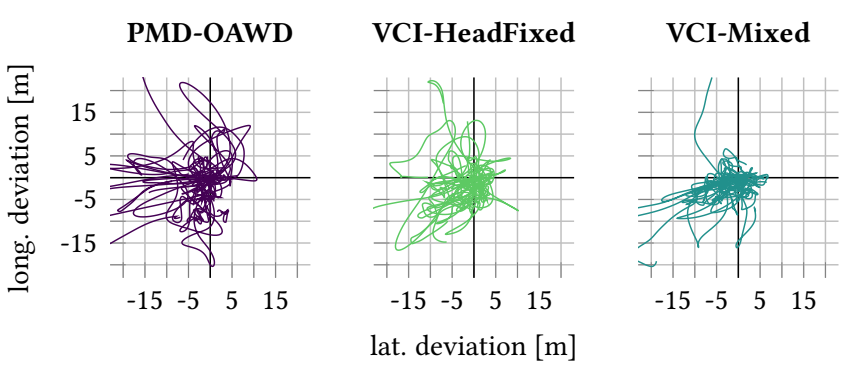

(a) Top view of the flight paths.

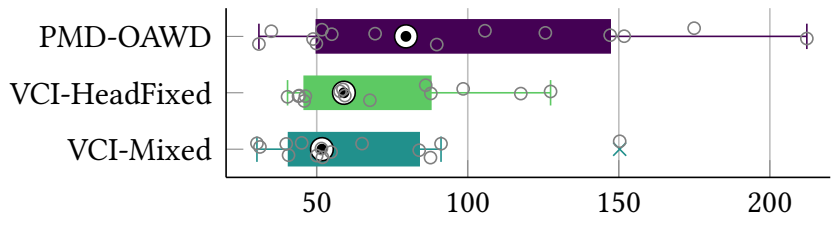

Distance covered during the hover phase $[\mathrm{m}]$

(b) Covered track.

Figure 13 - Positional accuracy during the $60 \mathrm{~s}$ hover phase of the JEDEYETM study II. ${ }^{2}$

pilots landed. In summary, all but two of the touchdown positions lie within one rotor radius around the middle of the helipad. This zone is depicted by the gray circle. Also, the longitudinal deviation is larger than the lateral position error. Comparing the display conditions, it appears that with the PFD-only head-up symbology all participants touched down before the desired landing spot. The PFD-VCI variant also seems to induce such behavior. In contrast, both conditions comprising the CLS show a nearly balanced spread in longitudinal direction.
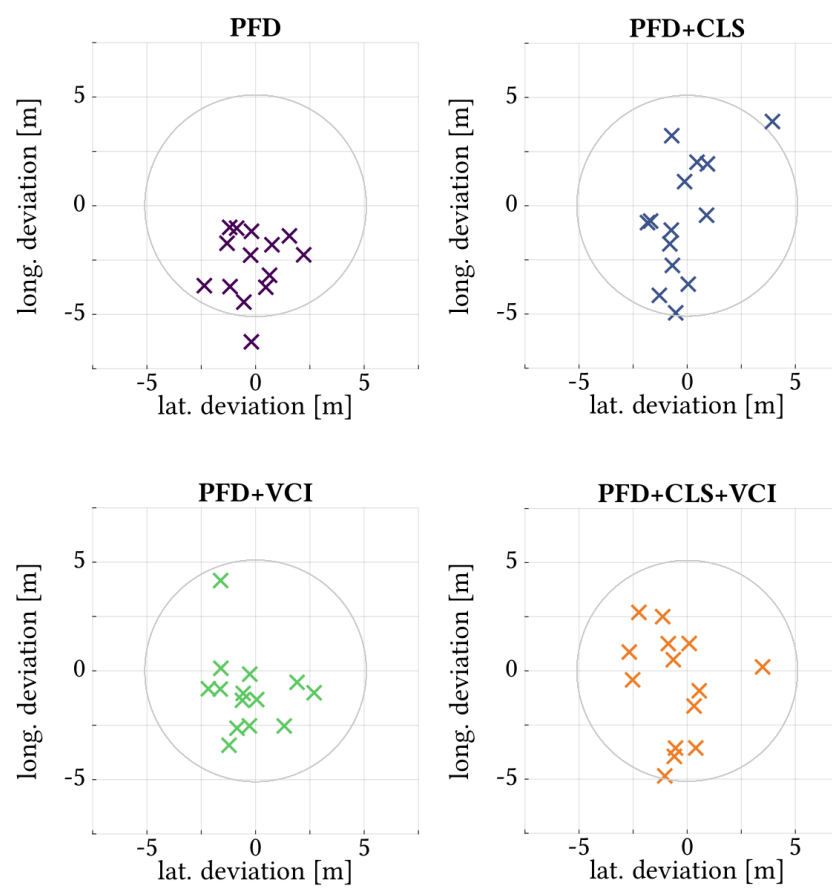

Figure 14 - Top views of the touchdown positions obtained with the four display variants. The graphs show lateral and longitudinal deviations from the desired landing spot in the middle of the platform. The gray circle illustrates the size of the rotor disk area.

An even more important criterion for a confined area landing is how well the pilot maintains a safe distance to nearby obstacles. Thus, we examined the minimum obstacle clear-

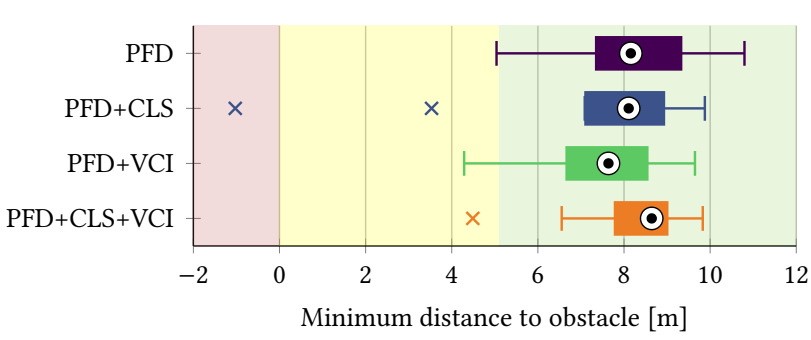

Figure 15 - Minimum obstacle clearance per flight grouped by display condition. ${ }^{1}$

ance recorded during each flight. Figure 15 depicts the results grouped by display condition. It can be seen that the majority of maneuvers was conducted with a safe obstacle clearance well above one rotor radius (> $5.1 \mathrm{~m}$; green zone). Nevertheless, during a few flights the required safety limit was undershot and even one collision occurred. The median and the middle $50 \%$ of the scores show no major difference between the tested symbology variants.

\subsection{Head Motion}

The idea behind a head-fixed VCI-OAWD was to enable the pilots to look out the window (and at the obstacles) more often than with a conventional PMD-based OAWD. The histograms in Fig. 16 indicate that this plan worked out. To see the wind turbine tower during the hover, the participants had to turn their head about $50^{\circ}$ to the right. The distribution of the head yaw rotations shows that - in all three display conditions - the pilots had two distinct areas of interest: in forward direction $\left(0^{\circ}\right)$ and in rightward direction (around $50^{\circ}$ ). However, the time spent in these areas varies significantly between the VCI positioning modes.

With AircraftFixed-HDD, the pilots' viewing direction was predominantly oriented straight ahead and rarely to the right where the wind turbine tower was located. This was expected since the VCI was located in forward direction, superimposed onto the instrument panel. Nevertheless, the pilots did not solely use the symbology but also - at times - looked to the obstacle on the right. In contrast, the two variants with the head-coupled VCI prompted the participants to make use of the VCI being always in sight: they looked much more to the right, where they could see both VCI and obstacle (cf. Fig. 2b).

Interestingly, with Mixed-HDD-Below the subjects hardly ever looked in directions between the two narrow areas of interest, whereas for HeadFixed-Below the gap between the peaks is less prominent and the right area of interest is considerably wider. This can be explained by the mode switching mechanism of the Mixed mode in the first study. The VCI switched from its aircraft-fixed head-down position to its head-fixed position if the pilot looked more than $23^{\circ}$ to the side. This caused the VCI to jump whenever the pilot's LOS crossed this limit. Thus, the subjects avoided this transition area. This behavior was changed for the Mixed mode in the second study: the position changes were not triggered by the pilot's LOS anymore but coupled to the flight phase.

In the second study, the limited simulation environment prevented us from observing this head motion behavior. The A350-like cockpit strongly restricted the pilots' out-thewindow view. Further, the outside vision projection system could not supply sufficient side-view. Therefore, the headmotion patterns were not as pronounced as during the VR study, which provided a much more realistic simulation of the pilot's view from a real helicopter cockpit. 
Aircraft-Fixed

HDD

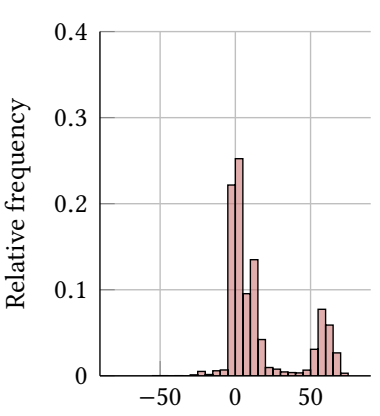

Mixed

HDD-Below

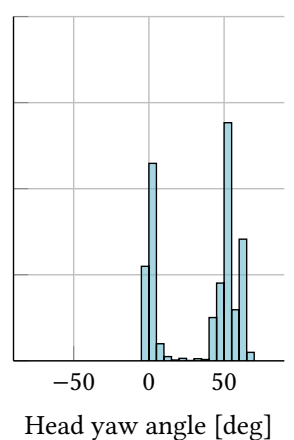

Head-Fixed

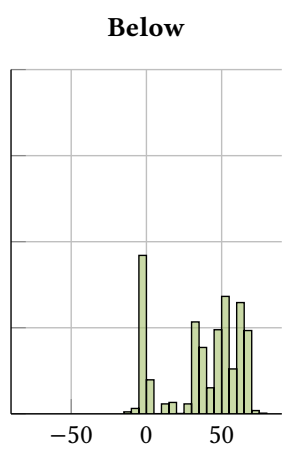

Figure 16 - Distribution of the pilots' head yaw rotation during the hover maneuver. Positive values correspond to viewing directions to the right where the wind turbine tower was located.

\section{Discussion of the Evaluation Results}

The subjective ratings clearly show that the pilots appreciate the advantages of a hover symbology in the form of a VCI. The positive results of the first study were confirmed by the more advanced second study with the JEDEYE ${ }^{\text {TM }}$ HMD. Both the head-fixed and the mixed VCI variant appeared to be helpful and the evaluation did not reveal major differences between the two VCI positioning modes. It seems to depend on personal preferences which one was favored by the participant. Nevertheless, the idea to couple the state of the VCI to the flight phase and the display content was perceived to be helpful. A possible conclusion from this result is that one could provide a number of feasible VCI options which can be selected depending on customer needs (defined in ConOps based on the task requirements). As mentioned in Sec. 3.1, this great flexibility is one of the major benefits of such virtual instruments.

In good visual conditions (study I), we did not find major differences in the achieved positional accuracy between the three pre-selected VCI modes. The flight performance was already good with the conventional AircraftFixed-HDD variant so that the head-fixed and mixed options could not significantly improve the position holding accuracy. Nevertheless, the head motion data reveals that both variants with head-coupled VCI allowed the pilots to keep their heads up and eyes out at the obstacles significantly more often. This is an important safety gain in such maneuvers even though this quantity is not directly measurable with our simulator study.

Due to the limited outside vision system of the GECO simulator, the second study showed the advantages that the VCI-OAWD creates if the out-the-window view is restricted. As expected, the limited visual cues degraded the overall hover performance considerably. Nevertheless, the setup also revealed the differences between the $\mathrm{VCI}$ variants and the baseline condition - where the OAWD was only displayed on the PMD.

A proven method to enhance the hover performance in DVE is to add acceleration cues to the display. However, such elements may capture a lot of attention and require excessive training before they can be used effectively. We did not include such a symbol set as our symbology does not target zero visibility conditions. It is devised as an addition to outside visual cues, not as a replacement. The VCI-OAWD should enable the pilot to keep the eyes out with the supplemen- tary OAWD always in sight. It should not fixate the pilot's attention on the symbology only. The pilots should not be animated to practice instrument-flying when they actually operate under visual flight rules (VFR).

Our research shows that the VCI appears to be a suitable alternative to a head-down OAWD. The pilots reported that it did not clutter their view but provided essential information in a user-friendly manner. A major plus is that the VCI allows the pilot to conduct the hover maneuver "head-up, eyes-out" while simultaneously monitoring the 360-deg obstacle awareness display. However - at the current stage - we have not experimentally compared this approach to spatial auditory or haptic cueing. Every modality has individual strengths and weaknesses when serving as an OAWD. For instance, a visual display with a top-down view seems to be most powerful in providing precise distance presentation and an overview of a complex environment, e.g. with several widespread obstacles. A spatial audio warning, on the other hand, might be better suited to indicate a high-priority obstacle. It can intuitively and immediately guide the pilot's attention to an urgent threat location which might be outside of the pilot's current FOV. The human auditory distance perception is rather inaccurate, which is why pulse-period cueing is often used to synthesize distance (cf. parking assistants in modern cars) [12]. Additionally, haptic feedback via active inceptors/sticks can directly guide the pilot's control inputs. However, it does not provide the strategic overall picture of the situation like a visual top-down view. In our opinion, auditory and haptic cues can potentially enhance but not replace the VCI. The ultimate solution will be a multi-modal cueing system where the modalities complement each other. This claim is, for example, supported by Godfroy-Cooper et al. [31] who showed that an isomorphic spatial visual-auditory display was favored over visual-only and audio-only representations by helicopter pilots in DVE.

\section{Conclusions \& Outlook}

This paper presented DLR's concept of virtual cockpit instruments as an extension to state-of-the-art HWD symbology. The assumed benefits of the VCI approach could be confirmed with two evaluation studies. The developed VCIOAWD received high subjective ratings and was preferred over a conventional PMD-based OAWD. All variants that kept the VCI within the pilot's FOV could increase the head-up, eyes-out times significantly. Even though these head-coupled 
VCI implementations (Mixed, Head-Fixed) could not improve the hover accuracy in good visual conditions (compared to the Aircraft-Fixed variant), they considerably enhanced the performance when the outside vision was restricted. Nevertheless, it is important to note that the symbology was not designed for instrument-only flying but as an addition to the natural out-the-window view in visual meteorological conditions (VMC).

\subsection{Future Research Directions}

We are planning a flight campaign to confirm our current results with the JEDEYETM HWD in DLR's EC135 research helicopter. One important test point will be to reassert the promising results of our simulator experiment regarding clutter and VCI readability. The flight tests have to confirm that this is also the case under actual in-flight conditions (vibrations, more complicated light setting, etc.).

Our exemplary implementation - the VCI-OAWD - provides a complementary top view without drawing the pilot's attention to a panel display inside the cockpit. This is also relevant in scenarios other than helicopter offshore operations. For instance, off airfield landings at unprepared sites seem to be an interesting scenario where the advantages of the VCI-OAWD could aid the pilot's spatial awareness. Moreover, the VCI-OAWD is not the only possible implementation of the VCI concept and this approach is not restricted to the helicopter domain. Thus, it will be interesting for future studies to explore other scenarios where VCIs can generate benefits. By doing so, one should keep in mind that the usefulness of VCIs is rather application dependent. For certain flight tasks the described advantages will not have any effect because such a flexible display setup is simply not needed in this case. During other maneuvers like the one presented here, however, a VCI can reasonably assist the pilot and help to increase flight safety.

In light of visual channel overload and HWD clutter, future research should also compare the VCI approach to haptic and spatial auditory displays. As discussed above, related work supports the hypothesis that a multi-modal cueing system can enhance the OAWS, especially for more complex obstacle scenarios, which the VCI will be applied to in the future. In our subsequent work, we plan to investigate if these results from related work can be transferred to our VCI approach.

With respect to the current technological advancements, we can assume that future HWDs will further promote the virtualization of cockpit instruments. For this work, we used a monochrome, military HMD, which was built as a prototype about 10 years ago. Today's and tomorrow's HWDs will offer even more opportunities for HMI designers. For instance, the availability of color as well as better display resolutions will enable the development of more advanced VCIs. Also, reduced acquisition costs and increased wearing comfort will make HWDs interesting for an application outside of the military sector, which offers new use cases for VCIs.

In the long term, we are planning to advance the VCI approach to a holistic cockpit concept. As a first step, we want to enable the pilots to interact with the virtual cockpit environment. The users should be able to adapt the VCI and choose options like they do with conventional instruments. Finally, it will be interesting to explore how far such a virtualization can go: Will it someday make sense to replace major parts of the physical HMI by a virtual version of a flight deck? DLR's research on the "Virtual Cockpit" [32] tries to examine such questions.

\subsection{Avionics Integration and Path to Certification}

Basically, an OAWS consists of a sensing, a processing, and a display subsystem. Our work focuses on the latter comprising graphics processing hardware, a head-tracking system, and the HWD with its peripheral hardware. For our prototypical implementation of the display subsystem, we used actual avionics hardware: the Elbit JEDEYETM as display unit (incl. head-tracking) and an industrial graphics platform from our project partner Diehl Aerospace Systems as graphics generation unit. Regarding the obstacle sensing and data processing, Leonardo already offers an EASA-certified system. Their OPLS meets the range and accuracy requirements of the selected offshore scenarios (see Sec. 2.2 for details). For the realization of a VCI-based OAWS, one could basically keep their sensor and processing units but replace the PMD by the HWD avionics described above. Our work successfully evaluates the VCI on state-of-the-art avionics components in a high-fidelity simulator (technology readiness level 5/6). As a next step, we plan to demonstrate the usability of the system in flight tests.

Currently, none of the available synthetic/enhanced/combined vision systems allows for additional operational credit like reduced minima. Nevertheless, many are certified and in service as assistance systems which support the crew's situation awareness, reduce workload and ultimately improve safety. This applies also to Leonardo's OPLS. The only type of vision system that currently extends operational capabilities is an enhanced flight vision system (EFVS) for certain fixed-wing approaches; a special form of enhanced vision system (EVS) with superimposed flight guidance symbology on a HUD [33]. HWDs have been mainly used by military pilots but they become increasingly available in the civil domain. Recently, the ClearVision ${ }^{\mathrm{TM}}$ EFVS with the SkyLens ${ }^{\mathrm{TM}}$ HWD by Universal Avionics was certified as the first EFVS with an HWD instead of a conventional HUD [34].

The described developments are encouraging and recent efforts in working groups like the RTCA SC-213 and the EUROCAE WG-79 show that vision system topics are under active development in the standardization and regulatory organizations. We cannot predict how long the path to a possible certification of such systems with operational credit will be. Nevertheless, we can suppose that it will be a stepwise expansion of operational capabilities. First, the OAWS has to prove its usefulness in terms of safety increase under current regulations without operational benefit. Thereafter, an incremental introduction of additional permissions for certain scenarios might be discussed.

\section{References}

[1] J. L. Tchon and T. J. Barnidge, "Review of the evolution of display technologies for next-generation aircraft," in Proc. SPIE Display Technologies and Applications for Defense, Security, and Avionics, 2015.

[2] J. E. Melzer, "Head-Mounted Displays," in Digital Avionics Handbook, C. R. Spitzer, U. Ferrell, and T. Ferrell, Eds., CRC Press, 2014.

[3] B. Foote and J. Melzer, "A history of helmet mounted displays," in Proc. SPIE Head- and Helmet-Mounted Displays, 2015. 
[4] H.-U. Döhler, J. M. Ernst, and T. Lüken, "Virtual aircraftfixed cockpit instruments," in Proc. SPIE Degraded Visual Environments: Enhanced, Synthetic, and External Vision Solutions, 2015.

[5] L. Ebrecht, J. M. Ernst, H.-U. Döhler, and S. Schmerwitz, "Integration of an Exocentric Orthogonal Coplanar 360 Degree Top View in a Head Worn See-Through Display Supporting Obstacle Awareness for Helicopter Operations," in Human Interface and the Management of Information, 2018.

[6] J. M. Ernst, L. Ebrecht, and S. Schmerwitz, "Virtual cockpit instruments displayed on head-worn displays - Capabilities for future cockpit design," in Proc. IEEE Digital Avionics Systems Conference (DASC), 2019.

[7] L. Ebrecht, J. M. Ernst, and S. Schmerwitz, "Virtual cockpit instruments and visual conformal symbology on head-worn displays for helicopter offshore landings," in Proc. 76th Vertical Flight Society International Annual Forum, 2020.

[8] J. M. Ernst and L. Ebrecht, "Virtual cockpit instruments on head-worn displays for helicopter offshore operations in confined areas," in Proc. IEEE Digital Avionics Systems Conference (DASC), 2020.

[9] J. M. Ernst, S. Schmerwitz, T. Lueken, and L. Ebrecht, "Designing a virtual cockpit for helicopter offshore operations," in Proc. SPIE Degraded Environments: Sensing, Processing, and Display, 2017.

[10] M. Brunetti, “The Guardian project: Reasons, concept and advantages of a novel obstacle proximity LIDAR system," in Proc. 40th European Rotorcraft Forum, 2014.

[11] T. Waanders, R. Scheiblhofer, Q. Qian, B. van Noort, V. Ziegler, F. Schubert, and R. Koerber, "Helicopter rotorstrike alerting system," in Proc. 41st European Rotorcraft Forum, 2015.

[12] J. D. Miller, M. Godfroy-Cooper, and Z. P. Szoboszlay, "Augmented-Reality Multimodal Cueing for Obstacle Awareness: Towards a New Topology for Threat-Level Presentation," in Proc. 75th American Helicopter Society International Annual Forum, 2019.

[13] N. Peinecke, S. Schmerwitz, H.-U. Döhler, and T. Lüken, "Review of conformal displays: more than a highway in the sky," Optical Engineering, vol. 56, no. 5, 2017.

[14] B. Lorenz, H. Többen, and S. Schmerwitz, "Human performance evaluation of a pathway HMD," in Proc. SPIE Enhanced and Synthetic Vision, 2005.

[15] J. E. Melzer, "HMDs as enablers of situation awareness: the OODA loop and sense-making," in Proc. SPIE Headand Helmet-Mounted Displays, 2012.

[16] Z. P. Szoboszlay, W. B. Albery, T. S. Turpin, and G. M. Neiswander, "Brown-Out Symbology Simulation (BOSS) on the NASA Ames Vertical Motion Simulator," in Proc. 64th American Helicopter Society International Annual Forum, 2008.

[17] B. T. Fujizawa, Z. P. Szoboszlay, M. P. R. Flanigen, M. J. S. Minor, M. Z. G. Morford, and B. M. Davis, "Degraded Visual Environment Mitigation Program NATO Fligth Trials: U.S. Army Flight Test and Results," in Proc. 43rd European Rotorcraft Forum, 2017.
[18] K. A. Feltman, K. Bernhardt, and A. Hayes, "Comparison of Two Visual Symbology Sets in Rotary-Wing Aircraft," in Proc. 75th American Helicopter Society International Annual Forum, 2019.

[19] Z. P. Szoboszlay, B. T. Fujizawa, C. R. Ott, J. C. Savage, S. M. Goodrich, R. A. McKinley, and J. R. Soukup, "3DLZ Flight Test of 2013: Landing an EH-60L Helicopter in a Brownout Degraded Visual Environment," in Proc. 70th American Helicopter Society International Annual Forum, 2014.

[20] T. Münsterer, T. Schafhitzel, M. Strobel, P. Völschow, S. Klasen, and F. Eisenkeil, "Sensor-enhanced 3D conformal cueing for safe and reliable HC operation in DVE in all flight phases," in Proc. SPIE Degraded Visual Environments: Enhanced, Synthetic, and External Vision Solutions, 2014.

[21] P. Völschow, T. Münsterer, M. Strobel, and M. Kuhn, "Display of real-time 3D sensor data in a DVE system," in Proc. SPIE Degraded Visual Environments: Enhanced, Synthetic, and External Vision Solutions, 2016.

[22] M. Zimmermann, M. Gestwa, C. König, J. Wolfram, S. Klasen, and A. Lederle, "First results of LiDAR-aided helicopter approaches during NATO DVE-mitigation trials," CEAS Aeronautical fournal, vol. 10, no. 3, 2019.

[23] S. Schmerwitz, T. Lüken, H.-U. Döhler, N. Peinecke, J. M. Ernst, and D. da Silva Rosa, "Conformal displays: human factors analysis of innovative landing aids," Optical Engineering, vol. 56, no. 5, 2017.

[24] F. Viertler and M. Hajek, "Evaluation of Visual Augmentation Methods for Rotorcraft Pilots in Degraded Visual Environments," fournal of the American Helicopter Society, vol. 62, no. 01, 2017.

[25] N. A. Stanton, K. L. Plant, A. P. Roberts, C. K. Allison, and M. Howell, "Seeing through the mist: an evaluation of an iteratively designed head-up display, using a simulated degraded visual environment, to facilitate rotary-wing pilot situation awareness and workload," Cognition, Technology \& Work, 2019.

[26] C. D. Wickens, "The When and How of Using 2-D and 3-D Displays for Operational Tasks," Proceedings of the Human Factors and Ergonomics Society Annual Meeting, vol. 44, no. 21, 2000.

[27] Brunner Elektronik AG, CLS-P Active Force Feedback Cyclic, Collective, Rudder, www.brunnerinnovation.swiss/produkt-uebersicht/, 2017.

[28] S. Greiser, R. Lantzsch, J. Wolfram, J. Wartmann, M. Müllhäuser, T. Lüken, H.-U. Döhler, and N. Peinecke, "Results of the pilot assistance system 'Assisted LowLevel Flight and Landing on Unprepared Landing Sites' obtained with the ACT/FHS research rotorcraft," Aerospace Science and Technology, vol. 45, 2015.

[29] Rotorsim, EC 135 Pro, www.rotorsim.de/ec135pro, 2019.

[30] O. Kreylos, The Display Resolution of Head-mounted Displays, Revisited, http://doc-ok.org/?p=1694, 2018.

[31] M. Godfroy-Cooper, J. D. Miller, E. Bachelder, and E. M. Wenzel, "Isomorphic Spatial Visual-Auditory Displays for Operations in DVE for Obstacle Avoidance," in Proc. 44th European Rotorcraft Forum, 2018. 
[32] J. M. Ernst, N. Peinecke, L. Ebrecht, S. Schmerwitz, and H.-U. Döhler, "Virtual Cockpit: an immersive headworn display as human-machine interface for helicopter operations," Optical Engineering, vol. 58, no. 5, 2019.

[33] Federal Aviation Administration, Enhanced Flight Vision Systems: AC 90-106A CHG 1, 2018.

[34] Elbit Systems Ltd., Universal Avionics Announces Certification for ClearVision ${ }^{T M}$ EFVS, https://elbitsystems.com/pr-new/universal-avionicsannounces-certification-for-clearvision-efvs/, 2020. 